\title{
The effect of fruit and vegetable consumption on physical function and muscle strength in older adults: the Ageing and Dietary Intervention Trial (ADIT)
}

\author{
C. E. Neville ${ }^{1}$, I. S. Young ${ }^{1}$, S. E. C. M. Gilchrist ${ }^{1}$, M. C. McKinley ${ }^{1}$, A. Gibson ${ }^{1}$, T. Banerjee ${ }^{2}$, \\ J. D. Edgar ${ }^{3}$ and J. V. Woodside ${ }^{1}$ \\ ${ }^{1}$ Nutrition and Metabolism Research Group, Centre for Public Health, Queen's University Belfast, Belfast, BT12 6BJ UK, \\ ${ }^{2}$ Unilever R\&D, Colworth Science Park, Sharnbrook, Bedfordshire, MK44 ILQ and ${ }^{3}$ Regional Immunology Service, \\ Belfast Health and Social Care Trust, Belfast, BT12 6BN, UK
}

Ageing is associated with numerous anatomical and physiological changes which can adversely affect both physical function and muscle strength, thus contributing to an increased risk of falls, fractures and disability. A growing body of observational evidence suggests that fruit and vegetable (FV) intake, which is often low in older people, and/or nutrients associated with a diet high in FV may protect against age-related decline in physical function and muscle strength ${ }^{(1-5)}$. However, this hypothesis has not yet been fully tested in intervention studies involving free-living older adults. The aim of the present study was to examine the effect of increased FV consumption on measures of muscle strength and physical function among healthy, free-living older adults.

A randomized controlled intervention study was undertaken. Eighty-three participants, aged 65 years and older and habitually consuming $\leq 2$ portions of FV/day, were randomised to continue their normal diet ( $\leq 2$ portions/day), or to consume $\geq 5$ portions of FV/day for 16 weeks. FV were delivered to all participants each week, free of charge. Compliance was monitored at baseline, 6 weeks, 12 weeks and 16 weeks by diet history and by measuring biomarkers of micronutrient status. Grip strength was measured by a hand-held dynamometer, while lower-extremity physical function was assessed by performance-based measures. Ethical approval for the study was obtained from the Office for Research Ethics Committees Northern Ireland (ORECNI).

Eighty-two participants completed the intervention. At week 16, the 5 portions/day group showed greater change in daily FV consumption compared to the 2 portions/day group $(P<0.001)$. The change in vitamin $\mathrm{C}$, lutein, zeaxanthin, $\beta$-cryptoxanthin and lycopene over the 16 weeks also differed significantly between groups, being higher in the 5/day group. No significant differences were evident in change in physical function between the two groups. However, there was a trend towards a greater change in grip strength in the 5 portions/day compared to the 2 portions/day group $(P=0.06)$.

\begin{tabular}{|c|c|c|c|c|c|c|c|c|c|c|c|c|}
\hline & \multicolumn{6}{|c|}{2 portions/day $\left(n_{\max }=39\right)$} & \multicolumn{6}{|c|}{5 portions/day $\left(n_{\max }=41\right)$} \\
\hline & \multicolumn{2}{|c|}{ Baseline } & \multicolumn{2}{|c|}{ Week 16} & \multicolumn{2}{|c|}{ Change at week $16^{\mathrm{a}}$} & \multicolumn{2}{|c|}{ Baseline } & \multicolumn{2}{|c|}{ Week 16} & \multicolumn{2}{|c|}{ Change at week $16^{\mathrm{a}}$} \\
\hline & Mean & $\overline{\mathrm{SD}}$ & Mean & $\mathrm{SD}$ & Mean & SD & Mean & SD & Mean & SD & Mean & $\mathrm{SD}$ \\
\hline Grip strength $(\mathrm{kg})$ & $24.2^{\mathrm{b}}$ & 8.1 & 24.3 & 8.5 & 0.1 & 3.3 & 33.2 & 10.3 & 35.2 & 10.6 & 2.0 & 5.2 \\
\hline Walk speed (sec) & 3.6 & 1.5 & 3.8 & 1.6 & 0.1 & 1.5 & 3.7 & 2.1 & 3.6 & 1.4 & -0.1 & 1.5 \\
\hline Chair stand (sec) & 11.5 & 4.3 & 11.5 & 4.7 & 0.0 & 3.6 & 10.8 & 4.6 & 10.2 & 4.5 & -0.6 & 4.0 \\
\hline Balance $(n): c$ & & & & & & & & & & & & \\
\hline Score 1 & $0^{\mathrm{d}}$ & & 0 & & & & 2 & & 0 & & & \\
\hline Score 2 & 1 & & 3 & & & & 1 & & 1 & & & \\
\hline Score 3 & 3 & & 3 & & & & 2 & & 4 & & & \\
\hline Score 4 & 35 & & 33 & & & & 36 & & 36 & & & \\
\hline SPPB scoree & 9.1 & 2.1 & 9.0 & 1.9 & -0.1 & 2.0 & 9.4 & 2.3 & 9.5 & 1.9 & 0.1 & 1.6 \\
\hline
\end{tabular}

${ }^{\mathrm{a}}$ Change calculated as wk 16-baseline. ${ }^{\mathrm{b}}$ Significant difference between groups at baseline $\left(P<0.001\right.$, independent samples t-test). ${ }^{\mathrm{c}} \mathrm{Scores}$ range from $1-4$ where 1 represents poor balance, 4 represents best balance. ${ }^{\mathrm{d}}$ Values are number of participants (all such values). ${ }^{\mathrm{e}} \mathrm{SPPB}=$ Short physical performance battery score $=$ sum of standing balance score + walk speed score + chair stand score. SPPB score ranges from 3-12.

Increased FV consumption had no effect on physical function. FV may enhance muscle strength in older adults however this observation needs to be confirmed in further intervention studies.

1. Cesari M, Pahor M, Bartali B et al. (2004) Am J Clin Nutr 79, 289-94.

2. Houston DK, Stevens J, Cai J et al. (2005) Am J Clin Nutr 81, 515-22.

3. Myint PK, Welch AA, Bingham SA et al. (2007) Public Health Nutr 10, 34-41.

4. Lauretani F, Semba RD, Bandinelli S et al. (2008) J Gerontol A Biol Sci Med Sci 63, 376-83.

5. Robinson SM, Jameson KA, Batelaan SF et al. (2008) J Am Geriatr Soc 56, 84-90. 